\title{
Evidence of tetragonal distortion as the origin of the ferromagnetic ground state in $\gamma$-Fe nanoparticles
}

\author{
V. Augustyns, ${ }^{1}$ K. van Stiphout, ${ }^{1}$ V. Joly, ${ }^{1}$ T. A. L. Lima, ${ }^{1}$ G. Lippertz, ${ }^{1}$ M. Trekels, ${ }^{1}$ E. Menéndez, ${ }^{1}$ F. Kremer, ${ }^{2}$ U. Wahl, ${ }^{3}$ \\ A. R. G. Costa, ${ }^{3}$ J. G. Correia, ${ }^{3}$ D. Banerjee, ${ }^{4}$ H. P. Gunnlaugsson, ${ }^{1}$ J. von Bardeleben, ${ }^{5}$ I. Vickridge, ${ }^{5}$ M. J. Van Bael, ${ }^{6}$ \\ J. Hadermann, ${ }^{7}$ J. P. Araújo,${ }^{8}$ K. Temst, ${ }^{1}$ A. Vantomme, ${ }^{1}$ and L. M. C. Pereira ${ }^{1, *}$ \\ ${ }^{1}$ KU Leuven, Instituut voor Kern - en Stralingsfysica, 3001 Leuven, Belgium \\ ${ }^{2}$ Department of Electronic Materials Engineering, Research School of Physics and Engineering, \\ The Australian National University, ACT 0200, Australia \\ ${ }^{3}$ Centro de Ciências e Tecnologias Nucleares, Instituto Superior Técnico, Universidade de Lisboa, 2695-066 Bobadela, Portugal \\ ${ }^{4}$ Dutch-Belgian Beamline (DUBBLE), ESRF - The European Synchrotron, CS 40220, 38043 Grenoble, France \\ ${ }^{5}$ Institut des Nanosciences de Paris (INSP), Universite Paris 6\&7, UMR 7588 au CNRS 140, 75015 Paris, France \\ ${ }^{6} \mathrm{KU}$ Leuven, Laboratory of Solid-State Physics and Magnetism, 3001 Leuven, Belgium \\ ${ }^{7}$ Electron Microscopy for Materials Science (EMAT), University of Antwerp, Groenenborgerlaan 171, 2020 Antwerp, Belgium \\ ${ }^{8}$ IFIMUP and IN-Institute of Nanoscience and Nanotechnology, Universidade do Porto, 4169-007 Porto, Portugal
}

(Received 9 June 2017; revised manuscript received 16 October 2017; published 7 November 2017)

\begin{abstract}
$\gamma$-Fe and related alloys are model systems of the coupling between structure and magnetism in solids. Since different electronic states (with different volumes and magnetic ordering states) are closely spaced in energy, small perturbations can alter which one is the actual ground state. Here, we demonstrate that the ferromagnetic state of $\gamma$-Fe nanoparticles is associated with a tetragonal distortion of the fcc structure. Combining a wide range of complementary experimental techniques, including low-temperature Mössbauer spectroscopy, advanced transmission electron microscopy, and synchrotron radiation techniques, we unambiguously identify the tetragonally distorted ferromagnetic ground state, with lattice parameters $a=3.76(2) \AA$ and $c=3.50(2) \AA$, and a magnetic moment of 2.45(5) $\mu_{B}$ per $\mathrm{Fe}$ atom. Our findings indicate that the ferromagnetic order in nanostructured $\gamma$-Fe is generally associated with a tetragonal distortion. This observation motivates a theoretical reassessment of the electronic structure of $\gamma$-Fe taking tetragonal distortion into account.
\end{abstract}

DOI: 10.1103/PhysRevB.96.174410

\section{INTRODUCTION}

Metallic Fe in the face-centered-cubic (fcc) structure $(\gamma-\mathrm{Fe})$ and related alloys are model systems of the interplay between crystal structure, electronic structure, and magnetism [1-9]. A well-known example of this interplay is the Invar effect (anomalous thermal expansion), which originates from the thermally induced transition between different electronic structures which are associated with different lattice volumes and different magnetic ordering states (e.g., in $\mathrm{Fe}-\mathrm{Ni}$ alloys [1,2,6,7,10], Fe-Cu alloys [3], $\mathrm{Pd}_{3} \mathrm{Fe}$ [11], and $\mathrm{Mn}_{88} \mathrm{Ni}_{12}$ [12]). The different electronic states are associated with similar total energies, and therefore small perturbations (change in temperature, strain, etc.) can dramatically change how the different states are populated, thereby strongly affecting structural, transport, and magnetic properties. Various states have been theoretically investigated for $\gamma$-Fe, including ferromagnetic, antiferromagnetic, and nonmagnetic states [13-17]. These studies typically consider an isotropic variation in lattice parameter for the different electronic/magnetic states, thereby maintaining the fcc structure. Here, we experimentally demonstrate that the ferromagnetic state of $\gamma$-Fe nanoparticles is, in fact, associated with a tetragonal distortion of the fcc structure, shedding new light on the longstanding question regarding the ground state of $\gamma-\mathrm{Fe}[1,8]$.

Since bulk $\gamma$-Fe is thermodynamically stable only at high temperature (1043-1667 K), experimental research on $\gamma$-Fe

\footnotetext{
*lino.pereira@kuleuven.be
}

has mostly relied on ultrathin $\mathrm{Fe}$ films epitaxially grown on $\mathrm{Cu}$ [18-22] and to a lesser extent on other fcc metals (e.g., Pd [23] and $\mathrm{Rh}$ [8]). The $\gamma$ phase is stabilized by the similar lattice constant $a$ of fcc $\mathrm{Cu}$ and $\mathrm{Fe}$ (3.61 and $3.54 \AA$, respectively). The lattice mismatch induces an in-plane tensile strain (along the two in-plane dimensions) and, consequently, a tetragonal distortion which stabilizes a ferromagnetic ground state within the first few Fe monolayers [18-24]. The question which we address here is, what is the ground state of $\gamma-\mathrm{Fe}$ (structural and magnetic) when tensile strain is applied along all three dimensions? Previous work has focused on $\gamma$-Fe nanoparticles embedded in $\mathrm{Cu}$, which are typically antiferromagnetic [25-27], although ferromagnetic [28] and paramagnetic (likely antiferromagnetic with a Néel temperature below $1.8 \mathrm{~K}$ ) [29] states have also been reported. Typically it is assumed that the $\gamma$-Fe nanoparticles retain the fcc structure. This assumption appears to hold for antiferromagnetic $\gamma$-Fe nanoparticles, although a transition to a slightly tetragonally distorted phase has been observed below the Néel temperature [27]. For the ferromagnetic $\gamma$-Fe, on the other hand, it has remained unclear whether the nanoparticles exhibit a tetragonal distortion, similarly to thin films. More recently, Baker et al. proposed that $\gamma$-Fe nanoparticles embedded in $\mathrm{Cu}_{1-x} \mathrm{Au}_{x}$ indeed develop a tetragonal distortion with increasing Au concentration $x$, i.e., increasing lattice constant of the host matrix, and consequently of the tensile strain acting on the nanoparticles [30]. However, the extended x-ray absorption fine structure (EXAFS) data on which this work was based did not allow for an unambiguous identification of the tetragonal distortion [30]. Here, we show that $\gamma$-Fe nanoparticles embedded in $\mathrm{SrTiO}_{3}$ have a 
ferromagnetic ground state associated with a tetragonal distortion. Using $\mathrm{SrTiO}_{3}$ as a host matrix, which is also cubic but with a much larger lattice parameter $(3.905 \AA)$, provides sufficient separation between the $\mathrm{x}$-ray diffraction peaks of $\gamma$-Fe and of the host matrix, allowing us to unambiguously identify the tetragonal distortion. It is nevertheless an extremely challenging system from a characterization point of view (due to, e.g., the small size and limited amount of the nanoparticles), requiring the use of low-temperature Mössbauer spectroscopy, advanced transmission electron microscopy, and synchrotron radiation techniques, in addition to more conventional experimental methods.

\section{METHODOLOGY}

\section{A. Sample preparation}

The samples consist of commercial $\mathrm{SrTiO}_{3}$ single-crystal substrates (Crystal $\mathrm{GmbH})$, [001] oriented, implanted with ${ }^{57} \mathrm{Fe}^{+}$ions to a fluence of $\sim 1 \times 10^{16}$ atoms per $\mathrm{cm}^{2}$, at $60 \mathrm{keV}$, at room temperature, and under an angle of $10^{\circ}$ to minimize ion channeling during implantation. Based on SRIM2008 simulations [31], we estimate a projected range $R_{p}=314 \AA$, straggling $\Delta R_{p}=140 \AA$, and an Fe-to-Ti peak concentration of approximately $22 \%$. Thermal annealing was subsequently performed under vacuum $\left(\sim 1 \times 10^{-5} \mathrm{mbar}\right)$, first at $500^{\circ} \mathrm{C}$ and then at $900^{\circ} \mathrm{C}$ (both for $10 \mathrm{~min}$ ). The first annealing step $\left(500^{\circ} \mathrm{C}\right.$ with a ramp rate of $\left.1.2^{\circ} \mathrm{C} / \mathrm{s}\right)$ induces the recrystallization of the implanted layer (amorphous upon implantation), whereas the second $\left(900^{\circ} \mathrm{C}, 5^{\circ} \mathrm{C} / \mathrm{s}\right)$ increases the magnetization (after the $500^{\circ} \mathrm{C}$ annealing the magnetization is negligible). A detailed optimization of the implantation and annealing parameters will be reported elsewhere.

\section{B. Structural characterization}

\section{Transmission electron microscopy}

The samples for the electron microscopy studies were prepared using focused-ion-beam (FIB) milling and ion milling. High-angle annular dark-field scanning transmission electron microscopy (HAADF STEM) and energy-dispersive X-ray (EDX STEM) spectroscopy experiments were performed using a FEI Titan 80-300 "cubed" microscope equipped with a Super-X detector and operated at $200 \mathrm{kV}$. The results were recorded using probes with convergence semiangles in the 21-25 mrad range (with a probe size of about $1 \AA$ ). The probe current ranged between 100 and 200 pA. High-resolution transmission electron microscopy (HR-TEM) was performed using a FEI Tecnai G2 or a Jeol 2100F microscope, both operated at $200 \mathrm{kV}$.

\section{Synchrotron radiation $x$-ray diffraction}

The synchrotron radiation $\mathrm{x}$-ray diffraction (SR-XRD) measurements were performed at the Rossendorf (Helmholtz Zentrum Dresden-Rossendorf) BM20 beamline (at room temperature with a wavelength of $1.078 \AA$ ) and at the French CRG beamline BM02-D2AM (at room temperature with a wavelength of $1.1808 \AA$ ) at the European Synchrotron Radiation Facility (ESRF). The peaks in the spectra are fitted for every measured crystallographic direction ([002], [311], and [202]) with three different fit models (Gaussian, Gaussian/Lorentzian blend, and Voigt) using a free command-line self-contained MATLAB function peakfit.m (Version 7.45). We then take the average $2 \theta_{\gamma \text {-Fe }}$ value over the three fit models, giving one $2 \theta_{\gamma \text {-Fe }}$ value for each measured direction. These three $2 \theta_{\gamma}$-Fe values are then used to calculate the in-plane $(a)$ and out-of-plane (c) lattice constants, assuming equal in-plane lattice constants (along the [100] and [010] direction, i.e., $a=b$ ).

\section{Emission channeling}

Emission channeling (EC) makes use of the charged particles emitted by a radioactive isotope [32]. A sample was first implanted with stable ${ }^{56} \mathrm{Fe}$ to a fluence of $1.3 \times 10^{16}$ atoms per $\mathrm{cm}^{2}$ and subsequently coimplanted with radioactive ${ }^{59} \mathrm{Fe}$ (with a half-life $t_{1 / 2}=46$ days) to a fluence of $1 \times 10^{13} \mathrm{at} / \mathrm{cm}^{-2}$ by implanting the precursor isotope ${ }^{59} \mathrm{Mn}\left(t_{1 / 2}=4.6 \mathrm{~s}\right)$, which decays to ${ }^{59} \mathrm{Fe}$. The radioactive implantation was carried out at the online isotope separator facility ISOLDE at CERN. Angular-dependent emission yields of the $\beta^{-}$particles emitted during decay were measured at room temperature along three crystallographic directions ([100], [211], and [110]). Quantitative lattice location is provided by fitting the experimental patterns with theoretical ones (calculated using the many-beam formalism [32]) using the two-dimensional fit procedure outlined in Ref. [33].

\section{Extended $x$-ray absorption fine structure}

Fluorescence EXAFS experiments were performed at the $\mathrm{X}$-ray absorption spectroscopy station of the Dutch-Belgian Beamline (DUBBLE, BM26) at ESRF. The measurements were carried out at the $\mathrm{Fe} K$ edge $(7112 \mathrm{eV})$ at $25 \mathrm{~K}$. Background subtraction, data processing, and fitting made use of ATHENA and ARTEMIS from the IFEFFIT 1.2.11c package [34,35]. Ab initio calculations (feff8 [36]) were used to determine the backscattering amplitude and phase shifts of the single scattering (SS) paths.

\section{Magnetic characterization}

\section{SQUID magnetometry}

The magnetic characterization was performed using a superconducting quantum interference device (SQUID) magnetometer (LOT-Quantum Design SQUID-VSM MPMS3), following strict procedures in order to avoid measurement artefacts and external magnetic contributions. These procedures were developed based on statistically relevant tests, which allowed us to determine the practical limits of SQUID magnetometry for the detection of ferromagnetism under various sample preparation, processing, and handling conditions [37]. All measurements were carried out with in-plane applied magnetic field along the $\langle 100\rangle$ axis.

\section{Ferromagnetic resonance}

The ferromagnetic resonance (FMR) measurements were performed with a standard Bruker X-band (9-GHz) spectrometer using $100 \mathrm{kHz}$ field modulation and lock-in detection. This gives rise to first-derivative line shapes. The magnetic field range was $0-1.9 \mathrm{~T}$ and the spectra were measured in the temperature range from $4 \mathrm{~K}$ to room temperature. The 
sample size was of the order of $3 \times 4 \mathrm{~mm}^{2}$. The observed FMR spectrum could be well fitted with a Lorentzian line shape. The intensity of the FMR spectrum was obtained from a double integration.

\section{Conversion electron Mössbauer spectroscopy: Correlating structural and magnetic information}

The conversion electron Mössbauer spectroscopy (CEMS) measurements were performed at various temperatures between $5 \mathrm{~K}$ and room temperature using a ${ }^{57} \mathrm{Co}(39 \mathrm{mCi})$ source mounted on a velocity drive set for a velocity scale of approximately $\pm 12 \mathrm{~mm} / \mathrm{s}$. At room temperature, a parallelplate avalanche detector (PPAD) was used [38]. This detector uses acetone under 25 mbar as counting gas. The bottom electrode, connected to a negative bias voltage, was in contact with the sample while the top electrode was grounded. At low temperature, the sample was mounted on a cryostat $(\mathrm{Ox}-$ ford instruments, MICROHR2, microstat HiRes microscope cryostat). The Mössbauer measurements were recorded using three channeltrons from Dr. Sjuts Optotechnik GmbH (model KBL15RS) [39]. The isomer shift $(\delta)$ values and associated velocity scale were calibrated relative to a room-temperature spectrum for an $\alpha{ }^{57} \mathrm{Fe}$ thin film deposited on Si measured in the absence of an external magnetic field. The spectra were analyzed using the VINDA code [40].

\section{RESULTS}

This section is divided in two parts. First, we describe the basic structure and magnetic characterization of the $\mathrm{Fe}$

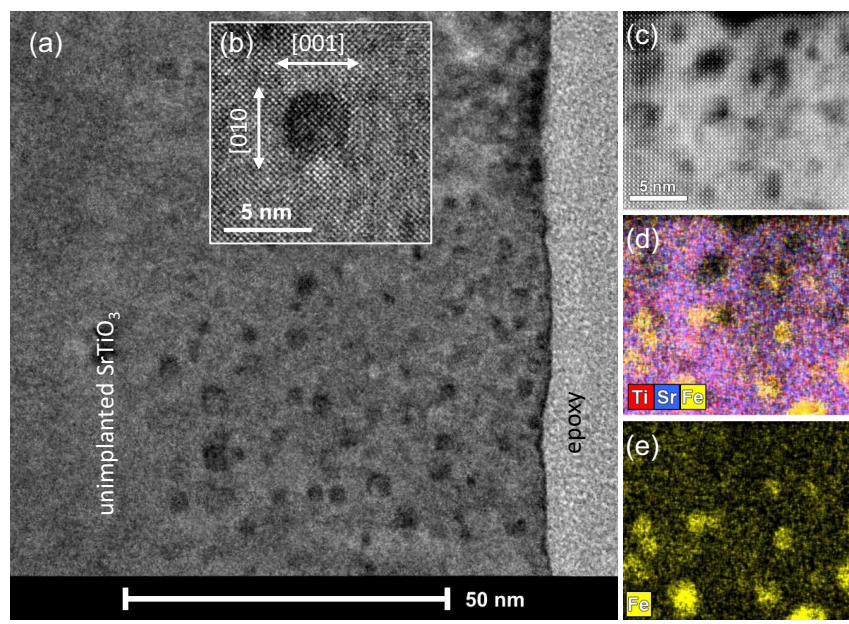

FIG. 1. Transmission electron microscopy (TEM) images taken along the [100] zone axis. (a) High-resolution TEM (HRTEM), low magnification, showing a near-surface layer of $\sim 70 \mathrm{~nm}$ with features of $2-5 \mathrm{~nm}$. (b) HRTEM, high magnification, on a $\gamma$-Fe nanoparticle with noticeable $\{100\}$ facets (although the atomic structure visible in the nanoparticle region is that of the $\mathrm{SrTiO}_{3}$ layer in which the particle is embedded). (c) High-angle annular dark-field (HAADF) scanning transmission electron microscopy (STEM). (d) Energy-dispersive X-ray (EDX) maps of the same region as (c), for $\mathrm{Ti}, \mathrm{Sr}$, and $\mathrm{Fe}$. (e) Same as (d) for Fe only. The EDX maps show that although most of the features observed in HRTEM can be ascribed to nanoparticles, some of them are also likely to be due to voids (large vacancy clusters).
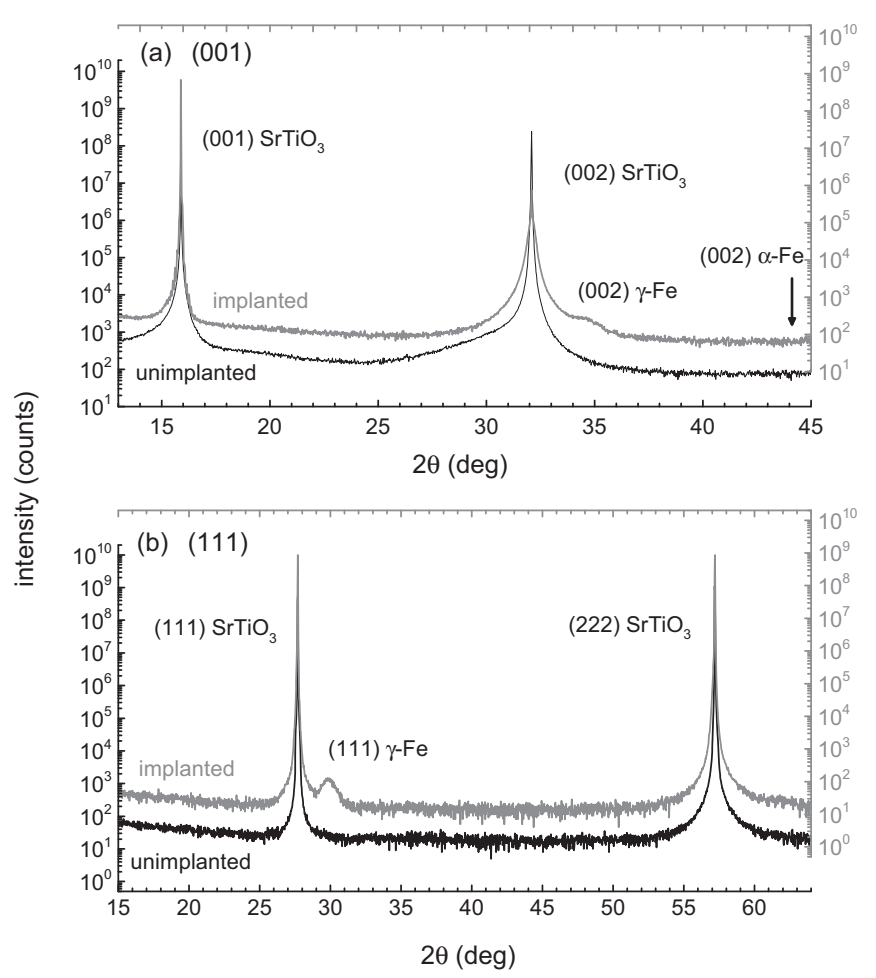

FIG. 2. Synchrotron radiation x-ray diffraction (SR-XRD) measured at room temperature with a wavelength of $1.078 \AA$. (a) Symmetric [001] and (b) asymmetric [111] $\omega / 2 \theta$ scans. For comparison, data for an unimplanted sample are also shown. In addition to the $\mathrm{SrTiO}_{3}$ substrate peaks, epitaxial $\gamma$-Fe peaks are observed, with pronounced peak broadening consistent with the small particle size $(2-5 \mathrm{~nm})$. The $2 \theta$ value for bulk $\alpha-\mathrm{Fe}(002)$ is indicated with a black arrow to emphasize that no $\alpha$-Fe is detected.

nanoparticles embedded in $\mathrm{SrTiO}_{3}$. In the second part we focus on the tetragonal distortion and its effect on the magnetic properties. From this point on, we refer to the tetragonally distorted structure as face-centered-tetragonal (fct). Although body-centered-tetragonal (bct) is a more accurate classification, fct is also widely used and in this case emphasizes that the observed structure is closer to fcc than it is to bcc.

After implantation and the two-step thermal annealing, the modified layer corresponds to the top $\sim 70 \mathrm{~nm}$ of the $\mathrm{SrTiO}_{3}$ substrate (TEM measurements in Fig. 1). Embedded in this layer, Fe nanoparticles with a diameter of approximately 5 nm can be observed using EDX spectroscopy [Fig. 1(e)]. Structurally, these Fe nanoparticles are identified as metallic $\mathrm{Fe}$ in the $\gamma$-Fe phase. Figure 2 shows the difference between unimplanted and implanted samples using SR-XRD, highlighting the presence of the $\gamma$-Fe phase in the SRXRD symmetric [001] and asymmetric [111] $\omega / 2 \theta$ scans. Additional asymmetric scans are discussed below, in the context of the fct distortion. The implanted layer (perovskite) and the $\gamma$-Fe nanoparticles exhibit an epitaxial relationship $(\langle 100\rangle||\langle 100\rangle$ and $\langle 111\rangle \|\langle 111\rangle)$, as evidenced by SR-XRD (Fig. 2), i.e., parallel $\{100\}$ planes of the implanted layer and $\gamma$-Fe, which is consistent with the $\{100\}$ facets observed by TEM for several of the $\gamma$-Fe nanoparticles [Fig. 1(b)]. We attribute the stability of the $\gamma$ phase in $\mathrm{SrTiO}_{3}(3.905 \AA)$ at 


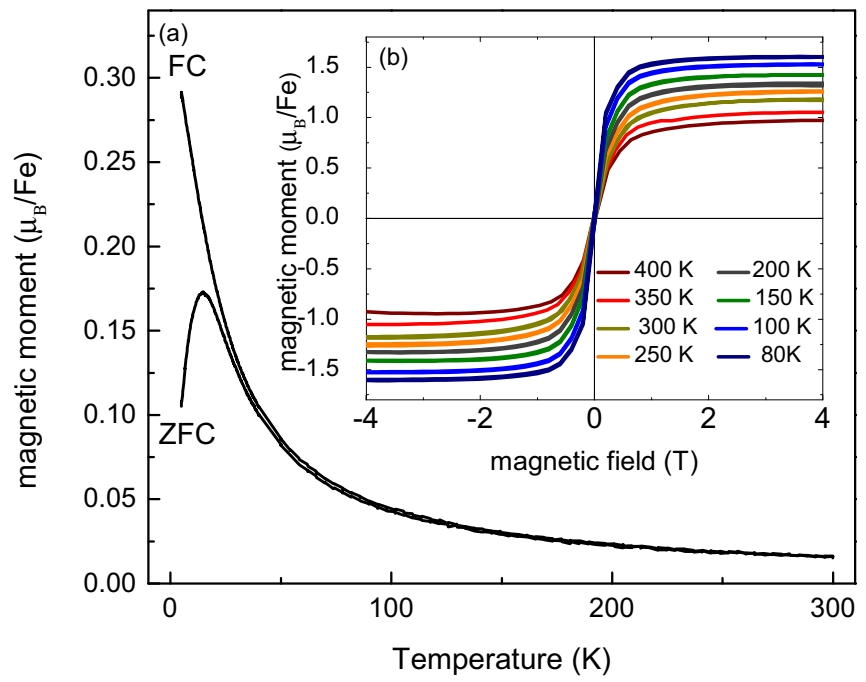

FIG. 3. SQUID magnetometry data with in-plane applied magnetic field along the [100] axis, expressed as magnetic moment in Bohr magnetons $\left(\mu_{B}\right)$ per Fe atom, normalized to all implanted Fe. (a) Field-cooled (FC) and zero-field-cooled (ZFC) measurements, with a field of $5 \mathrm{mT}(50 \mathrm{Oe})$. (b) Isothermal magnetization curves after subtraction of the diamagnetic component determined by a linear fit to the data in the high-field region (4-5 T).

room temperature to the better lattice matching of $\gamma$-Fe (8\% lattice mismatch) compared to bcc $(\alpha) \mathrm{Fe}(27 \%)$.

SQUID magnetometry measurements reveal a typical superparamagnetic behavior (Fig. 3), with a blocking temperature (relative to the magnetometry measurement time scale of seconds) of approximately $13 \mathrm{~K}$ [associated to the peak temperature in the field-cooled and zero-field-cooled magnetometry measurements in Fig. 3(a)], consistent with small nanoparticles (nm size) with a ferromagnetic ground state and a Curie temperature $\left(T_{C}\right)$ above $400 \mathrm{~K}$ (highest measured temperature). The FMR measurements exhibit broad line spectra characteristic of superparamagnetic particles (Fig. 4). When the temperature is lowered below room temperature, the FMR line broadens and shifts to lower magnetic fields. Below $200 \mathrm{~K}$ the resonance is no longer discernible due to excessive line broadening.

It is important to note that not all of the implanted Fe atoms precipitate into $\gamma$-Fe nanoparticles. A significant fraction of the implanted $\mathrm{Fe}$ substitutes for $\mathrm{Ti}$, forming a $\mathrm{Sr}(\mathrm{Ti}, \mathrm{Fe}) \mathrm{O}_{3}$ matrix in which the $\gamma$-Fe nanoparticles are embedded. The combination of EC, EXAFS, and XRD provides a detailed description of the nonprecipitated $\mathrm{Fe}$ component. Figure 5 shows ${ }^{59} \mathrm{Fe}$ EC data and the best fit, which is obtained for $34(8) \%$ of $\mathrm{Fe}$ atoms in $\mathrm{Ti}$ sites, with the remaining $66(8) \%$ contributing with an isotropic emission (in random sites). The random component can be attributed to ${ }^{59} \mathrm{Fe}$ present in $\gamma$-Fe nanoparticles and in $\mathrm{Sr}(\mathrm{Ti}, \mathrm{Fe}) \mathrm{O}_{3}$ regions which are either disordered or not epitaxially recrystallized. Since $\gamma$-Fe or disordered/nonepitaxial $\mathrm{Sr}(\mathrm{Ti}, \mathrm{Fe}) \mathrm{O}_{3}$ are not perfectly coherent with the epitaxial $\mathrm{Sr}(\mathrm{Ti}, \mathrm{Fe}) \mathrm{O}_{3}$ layer, the $\beta^{-}$particles emitted from $\mathrm{Fe}$ atoms within $\gamma$-Fe nanoparticles are more likely to be dechanneled, thereby contributing with an isotropic emission (cf. Ref. [41] for a more detailed discussion on these effects in Fe-implanted $\mathrm{ZnO}$ ). The coexistence of $\gamma$-Fe nanoparticles

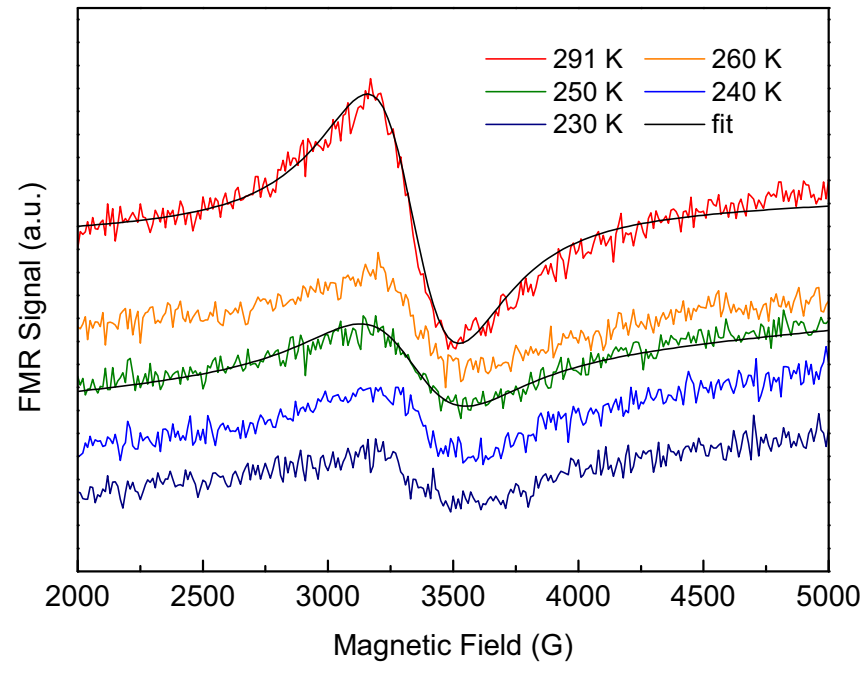

FIG. 4. FMR spectra as a function of temperature, with the magnetic field applied perpendicular to the sample plane, and fit (for 291 and $250 \mathrm{~K}$ ) with a Lorentzian line shape (black line).

and dilute Fe in Ti sites is also confirmed by EXAFS (Fig. 6), yielding fractions of 63(12)\% and 37(12)\% for $\mathrm{Fe}$ in $\gamma$-Fe and $\mathrm{Sr}(\mathrm{Ti}, \mathrm{Fe}) \mathrm{O}_{3}$, respectively. The nonprecipitated $\mathrm{Fe}$ fraction in $\mathrm{Sr}(\mathrm{Ti}, \mathrm{Fe}) \mathrm{O}_{3}$ exhibits Brillouin-like paramagnetic behavior (cf. Supplemental Material [42]).

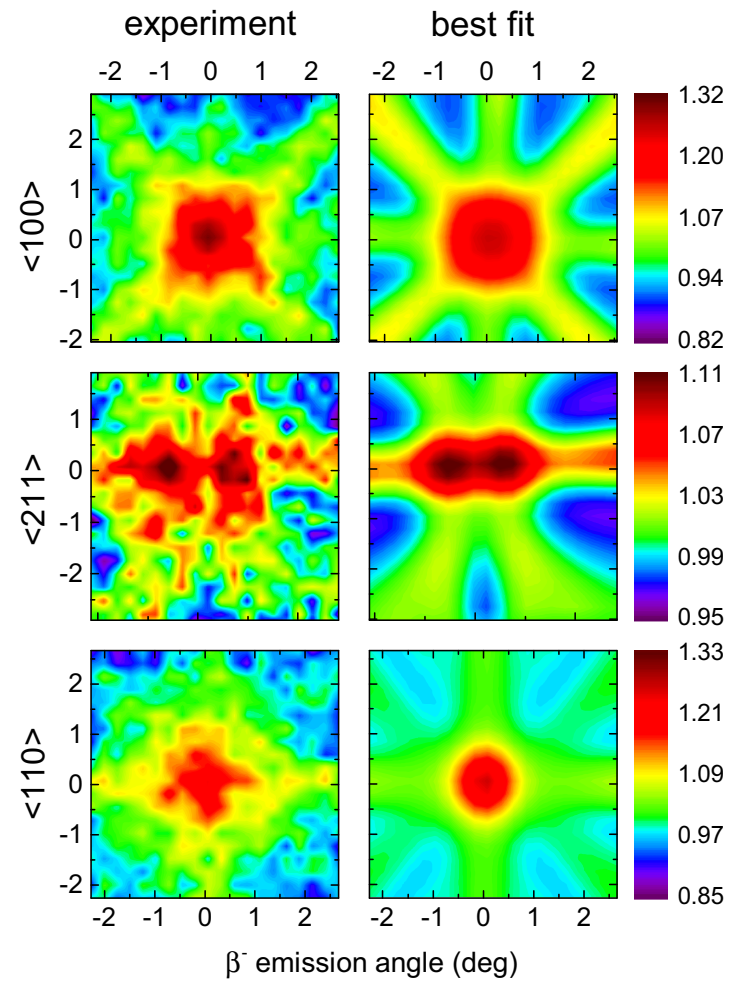

FIG. 5. (Left) Experimental ${ }^{59} \mathrm{Fe} \beta^{-}$emission channeling patterns in the vicinity of the $\langle 100\rangle,\langle 110\rangle$, and $\langle 211\rangle$ directions. (Right) Corresponding best fits yielding $34(8) \%$ of the Fe atoms in Ti sites, and the remaining 66(8)\% contributing with an isotropic emission (attributed to ${ }^{59} \mathrm{Fe}$ in $\gamma$-Fe nanoparticles and disordered/nonepitaxial regions). 


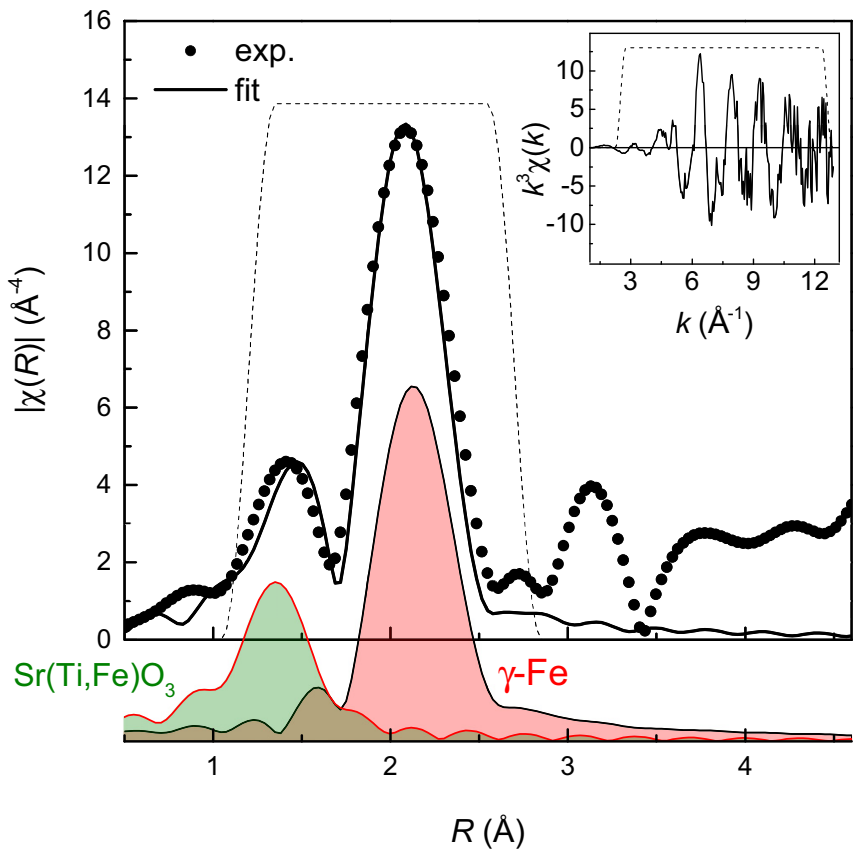

FIG. 6. Extended x-ray absorption fine structure (EXAFS) data measured in the vicinity of the Fe $K$ edge, at room temperature. (Top) Magnitude of the Fourier transform (symbols) as a function of non-phase-corrected radial distance: experimental data (symbols) and best fit (black line) allowing for two fractions of Fe atoms in $\mathrm{Ti}$ sites in $\mathrm{Sr}(\mathrm{Ti}, \mathrm{Fe}) \mathrm{O}_{3}$ and in $\gamma$-Fe. The dashed line represents the Hanning window from 1.2 to $2.7 \AA$ with a width of $0.3 \AA$ used in the fit. (The data corresponding to higher-order coordination shells is not included in the fit.) (Bottom) Magnitude of the Fourier transform corresponding to the first shell in $\mathrm{Sr}(\mathrm{Ti}, \mathrm{Fe}) \mathrm{O}_{3}$ and in $\gamma$-Fe, illustrating how the analysis discriminates between the two components. (Inset) Spectra of $k^{3}$-weighted EXAFS as a function of photoelectron momentum. The dashed line represents the Hanning window from 2 to $13 \AA^{-1}$ with a width of $0.5 \AA^{-1}$ used in the fit.

CEMS measurements at various temperatures between $5 \mathrm{~K}$ and room temperature (Fig. 7) confirm the presence of $\gamma$-Fe and nonprecipitated $\mathrm{Fe}\left[\mathrm{Sr}(\mathrm{Ti}, \mathrm{Fe}) \mathrm{O}_{3}\right]$ components. Details on the analysis and fitting model are given in the Supplemental Material [42]. $\gamma$-Fe appears as a mixture of two components: superparamagnetic and blocked $\gamma-\mathrm{Fe}$ nanoparticles, with a total $\gamma-\mathrm{Fe}$ fraction of $64.6(3) \%$. The ratio of superparamagnetic to blocked components increases with increasing temperature [Fig. 7(c)] due to superparamagnetic relaxation. Compared to magnetometry measurements, the shorter time scale associated with the Mössbauer state (98 ns) results in a higher blocking temperature $T_{b}$. A $T_{b}$ of $13 \mathrm{~K}$ for magnetometry measurements corresponds to a $T_{b}$ of approximately $30 \mathrm{~K}$ for CEMS measurements (assuming Néel relaxation [43]), which is consistent with the significant increase in superparamagnetic fraction between $10 \mathrm{~K}$ and $50 \mathrm{~K}$ [Fig. 7(c)]. The most important conclusion to be drawn from the CEMS measurements is that the isomer shift of the magnetic component corresponds to $\gamma$-Fe [Fig. 7(b)] [44-47], not $\alpha$-Fe or $\mathrm{Sr}(\mathrm{Ti}, \mathrm{Fe}) \mathrm{O}_{3}$. This unambiguously establishes that the superparamagnetic component in the magnetometry

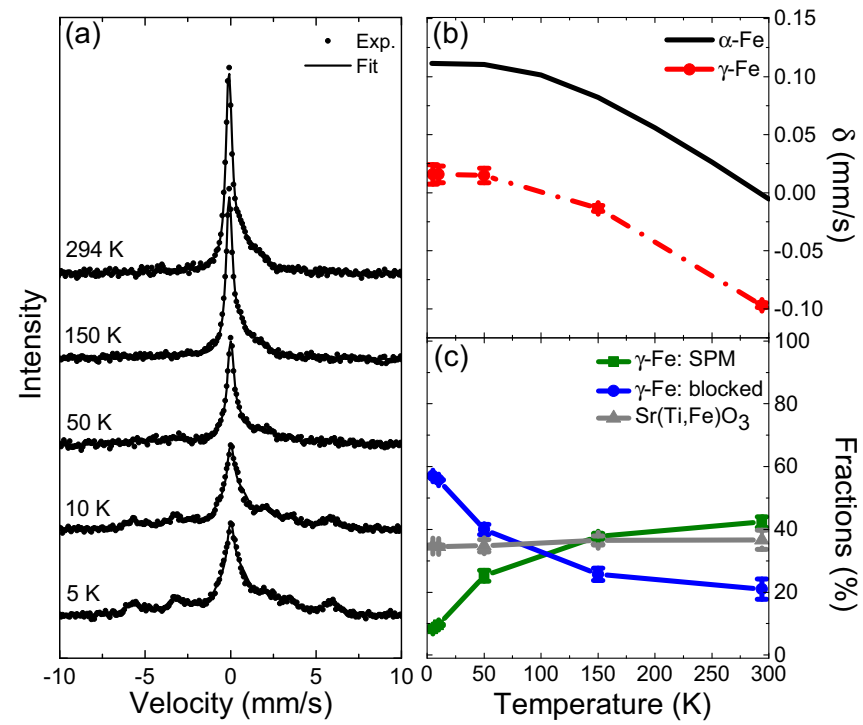

FIG. 7. (a) Conversion electron Mössbauer spectroscopy (CEMS) data and fit measured at different temperatures (5-294 K). (b) The measured isomer shift corresponding to $\gamma$-Fe, compared to that of $\alpha$-Fe, measured in the same setup on a $20-\mathrm{nm} \alpha-\mathrm{Fe}$ film deposited on Si. (c) The fractions of the different components: $\gamma-\mathrm{Fe}$ in the blocked regime, $\gamma$-Fe in the superparamagnetic (SPM) regime, nonprecipitated $\mathrm{Fe}$ in $\mathrm{Sr}(\mathrm{Ti}, \mathrm{Fe}) \mathrm{O}_{3}$ (the fit model is described in Supplemental Material [42]).

data originates from $\gamma$-Fe nanoparticles with a ferromagnetic ground state.

As in the work of Baker et al. [30], our EXAFS data do not allow us to identify unambiguously whether the $\gamma$-Fe nanoparticles have fcc or fct structure. The fit improvement obtained by allowing for an fct distortion is only marginal (cf. Supplemental Material [42]). On the other hand, SRXRD measurements along different crystallographic directions ([002], [311], and [202]) provide direct evidence of fct distortion. Figure 8 shows SR-XRD data measured in the vicinity of the [002] and [311] diffraction peaks of $\mathrm{SrTiO}_{3}$ and $\gamma-\mathrm{Fe}$, which are well separated, thanks to the significant difference in lattice parameter. Figure 8 also compares the data to the expected position of the diffraction peaks for bulk $\gamma$-Fe (fcc with $a=c=3.54 \AA$, based on extrapolation from antiferromagnetic Fe alloys [48]). The [002] direction is only sensitive to the out-of-plane lattice parameter $c$, whereas [311] and [202] depend on both $c$ and the in-plane lattice parameter $a$ (with the [311] direction being the most sensitive to changes in $a$ ). Combining the fit results for all three directions, we obtain $a=3.76$ (2) $\AA$ and $c=3.50$ (2) $\AA$ (the error includes contributions from the fit for each direction, from variations across the different directions and from the difference between measured and theoretical values of the $\mathrm{SrTiO}_{3}$ matrix), corresponding to an fct distortion $\epsilon_{\mathrm{fct}}=\frac{a-c}{a_{\text {bulk }}}=7.3(8) \%$. We can therefore conclude that the $\gamma$-Fe nanoparticles adopt an fct structure to accommodate the tensile strain imposed by the $\mathrm{Sr}(\mathrm{Ti}, \mathrm{Fe}) \mathrm{O}_{3}$ host matrix, similar to ultrathin $\gamma$-Fe films on fcc metals [8,18-23], despite the fact that in this case the strain is applied along all three dimensions as opposed to two dimensions in thin films. As in the thin-film case, this fct 


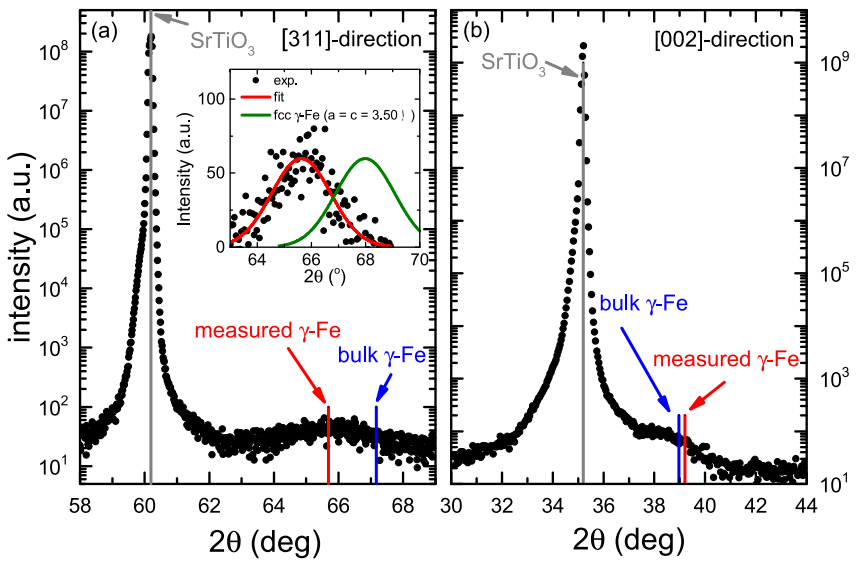

FIG. 8. SR-XRD measured at room temperature with a wavelength of $1.1808 \AA$. (a) Asymmetric (311) and (b) symmetric (002) $\omega / 2 \theta$ scans. The measured $2 \theta$ values obtained from the fit are indicated in red, whereas the $2 \theta$ values corresponding to bulk fcc $\gamma$-Fe are indicated in blue $(a=c=3.54 \AA$, based on extrapolation from Fe-alloy data [48]). The inset illustrates that the data cannot be reproduced by an expanded fcc structure instead of an fct distortion, by comparing the experimental data of (a) in the region of the (311) peak of $\gamma$-Fe peak (solid symbols) and the fit (red line) to that same fit shifted to the $2 \theta$ value corresponding to $a=c=3.50 \AA$ (green line), i.e., of an fcc lattice with the $c$ parameter determined from the (002) direction.

distortion stabilizes the ferromagnetic ground state (resulting in superparamagnetic behavior in the case of nanoparticles). The observation of a ferromagnetic ground state instead of an antiferromagnetic one for nanoparticles with a unit cell volume of $49.6(5) \AA^{3}$ is also consistent with the high-volume (48.22 $\AA^{3}$ ) ferromagnetic state extrapolated from ferromagnetic $\gamma$-Fe-based alloys, compared to the low-volume (44.36 $\AA^{3}$ ) antiferromagnetic state extrapolated from antiferromagnetic $\gamma$-Fe-based alloys [48]. We also observe that the fct distortion induces a magnetocrystalline anisotropy, which is visible in the magnetization data as in-plane versus out-of-plane anisotropy: a lower saturation field and higher thermoremanent magnetization for the in-plane field, corresponding to an inplane easy axis (Fig. 9). Taking the definition of the anisotropy field $H_{a}$ which is required to saturate the magnetization of a uniaxial crystal in a hard direction (of the order of a few $\mathrm{T}$ along the [001] direction in this case, Fig. 9), we can estimate a magnetocrystalline anisotropy constant $K_{u}$ of the order of $10^{6} \mathrm{~J} / \mathrm{m}^{3}$ using $K_{u}=H_{a} \mu_{0} M_{s} / 2$ [43], with $M_{s}$ being the saturation magnetization. Note that this is not the magnetic anisotropy component responsible for the blocking/relaxation behavior observed in the temperature-dependent magnetic measurements (magnetometry and CEMS). Taking the blocking temperature from magnetometry measurements $(13 \mathrm{~K})$, we estimate an anisotropy constant below $10^{5} \mathrm{~J} / \mathrm{m}^{3}$ (assuming Néel relaxation [43]), i.e., at least 1 order of magnitude below the magnetocrystalline anisotropy constant $\left(10^{6} \mathrm{~J} / \mathrm{m}^{3}\right)$. This weaker anisotropy component is most likely due to shape anisotropy originating from, e.g., the particles not being perfectly spherical but exhibiting some degree of faceting
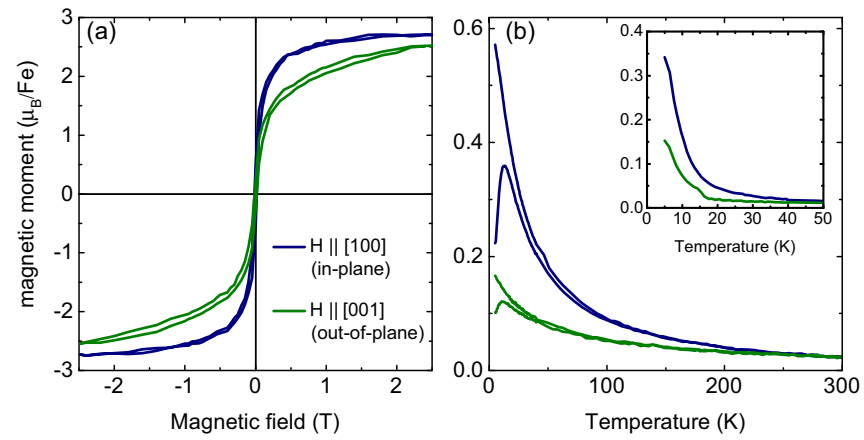

FIG. 9. SQUID magnetometry data measured with the magnetic field applied in-plane parallel to the [100] axis (blue) and out-of-plane parallel to the [001] axis (green), expressed as magnetic moment in Bohr magnetons $\left(\mu_{B}\right)$ per Fe atom in $\gamma$-Fe nanoparticles $(\gamma$-Fe fraction obtained from CEMS measurements). (a) Magnetization curves measured at $5 \mathrm{~K}$ after subtraction of the linear component determined by a linear fit to the data in the high-field region (2-3 T), corresponding to the diamagnetic substrate and the Brillouinlike paramagnetic Fe (dilute component). The saturation moment is slightly higher than that quoted in the text due to the small nonlinear contribution from the Brillouin-like paramagnetism of the dilute component at $5 \mathrm{~K}$. (b) FC and ZFC magnetization. Inset: Thermoremanent magnetization.

[Fig. 1(b)] or a prolate/oblate shape, which is consistent with an anisotropy constant of the order of $10^{5} \mathrm{~J} / \mathrm{m}^{3}$ [49].

Finally, we can determine the average moment per $\mathrm{Fe}$ atom in the $\gamma$-Fe nanoparticles by dividing the saturation moment by the number of $\mathrm{Fe}$ atoms in the $\gamma$ phase. For the total saturation moment of the $\gamma$-Fe component, we take the value at $40 \mathrm{~K}\left[3.64(2) \times 10^{-5} \mathrm{emu}\right]$, a sufficiently low temperature to be comparable to the truly intrinsic moment per $\mathrm{Fe}($ at $0 \mathrm{~K})$, but sufficiently high for the Brillouin-like paramagnetic magnetization of the dilute $\mathrm{Fe}$ component to be linear in field, i.e., being subtracted together with the diamagnetic background from the substrate (also linear in field). The number of $\mathrm{Fe}$ atoms in the $\gamma$ phase is obtained by multiplying the total number of implanted $\mathrm{Fe}$ atoms $\left(9.7 \times 10^{15} \mathrm{at} / \mathrm{cm}^{2}\right)$ by the corresponding fraction determined experimentally. As described above, different techniques (EC, EXAFS, and CEMS) were used here to quantify the fraction of $\mathrm{Fe}$ in the two components $[\gamma-\mathrm{Fe}$ nanoparticles versus dilute $\mathrm{Fe}$ in $\mathrm{Sr}(\mathrm{Ti}, \mathrm{Fe}) \mathrm{O}_{3}$ ]. Considering the data reported here, although all techniques yield equal fractions within error, CEMS [64.6(3)\%] gives the best precision. Taking the $\gamma$-Fe fraction obtained from CEMS, we obtain a moment per Fe atom in $\gamma$-Fe of 2.45(5) $\mu_{B}$.

\section{DISCUSSION}

We have established that $\gamma$-Fe nanoparticles embedded in $\mathrm{SrTiO}_{3}$ have an fct ferromagnetic ground state. We can now compare our findings $\left[\epsilon_{\mathrm{fct}}=7.3(8) \% ; 2.45(5) \mu_{B}\right.$ per $\mathrm{Fe}$ atom] to the recent work of Baker et al. suggesting that $\gamma-\mathrm{Fe}$ nanoparticles embedded in $\mathrm{Cu}_{1-x} \mathrm{Au}_{x}$ may be fct distorted [30]. Taking the nearest-neighbor Fe-Fe distance obtained from the fct fit to their EXAFS data, we obtain an $\epsilon_{\mathrm{fct}}$ of $5(2) \%$ for the highest Au concentration $(x=0.12)$, with an 
associated moment 2.5(2) $\mu_{B}$ per atom [30]. For lower $\mathrm{Au}$ concentration (i.e., smaller lattice parameter $\mathrm{Cu}_{1-x} \mathrm{Au}_{x}$ and therefore lower tensile strain) both the distortion and the moment per $\mathrm{Fe}$ decrease [30]. We can therefore conclude that $\gamma$-Fe nanoparticles embedded in $\mathrm{SrTiO}_{3}$ are similar to those embedded in $\mathrm{Cu}_{1-x} \mathrm{Au}_{x}$ in the limit of high tensile strain and associated fct distortion (similar $\epsilon_{\mathrm{fct}}$ and $\mu$ values). Remarkably, these similarities extend to $\gamma$-Fe thin films on $\mathrm{Cu}_{1-x} \mathrm{Au}_{x}: 2.6 \mu_{B}$ [50] and $2.7 \mu_{B}$ [51] for films with approximately the same in-plane lattice parameter $a(3.76 \AA)$, i.e., in the extreme of tensile strain. Showing that ferromagnetic $\gamma$-Fe has an fct structure, both in nanoparticle and thin-film form, provides a unifying picture of ferromagnetism in these systems. In particular, it suggests that the inconsistencies among reported ground states of $\gamma$-Fe nanoparticles [25-29] may be solved if the fct distortion is taken into account. One can expect that if fct structures are considered in theoretical assessments of the magnetic states of $\gamma$-Fe nanoparticles, the same degree of agreement between theory and experiment as achieved for ultrathin films [24] may also be reached. Some insight may already be obtained by considering recent density functional theory (DFT) calculations on bulk $\gamma$-Fe [52]. Various ordered and nonordered states are closely spaced in energy and have different dependencies on the magnitude of the fct distortion [52]. When the magnetic exchange energy is not taken into account, the local energy minimum coincides with the fcc structure. However, for the ordered states (ferromagnetic and antiferromagnetic), introducing an fct distortion decreases the total energy. In particular, in the region corresponding to the fct distortion reported here $(c / a$ of the order of 0.9 ), the ground state is indeed ferromagnetic. One can therefore expect that, if equivalent DFT calculations are performed for strained nanoparticles, these will reveal that the equilibrium lattice parameters (and therefore the magnitude of the fct distortion) are not only determined by the lattice parameters of the host lattice (conventional epitaxial strain), but that the exchange energy associated with ordered states also plays a crucial role.

fct distortion may in fact be a much more general phenomenon, extending to other observed magnetic ground states of $\gamma$-Fe. For example, $\gamma$-Fe nanoparticles in the low-volume antiferromagnetic state, with an fcc structure above the Néel temperature $\left(T_{N}\right)$, have been observed to exhibit a fcc-to-fct structural transition upon crossing $T_{N}$ (also a c-axis contraction as in the present case, although much smaller, 0.32\%) [27]. In an even wider context, hints of fct-related phenomena have emerged in the context of thermal expansion of Invar alloys. For example, anisotropic thermal expansion was observed in $\mathrm{Mn}_{88} \mathrm{Ni}_{12}$ : Invar effect for the $c$ parameter (abnormally small expansion) and anti-Invar (abnormally large) for the $a$ parameter [12]. Such puzzling observations further motivate a theoretical reassessment of the magnetic ground states of elemental $\gamma$-Fe taking into account fct distortions as a model for more complex alloys.

\section{CONCLUSIONS}

We can summarize our findings in three key observations: (i) 2-5-nm-sized $\gamma$-Fe nanoparticles were successfully embedded in $\mathrm{SrTiO}_{3}$; (ii) these $\gamma$-Fe nanoparticles exhibit a ferromagnetic ground state, unambiguously identified using low-temperature Mössbauer spectroscopy; (iii) the ferromagnetic ground state is associated with the fct structure, not fcc, as demonstrated by synchrotron radiation $\mathrm{x}$-ray diffraction measurements. These findings indicate that the fct structure underlies a universal relation between different forms of magnetically ordered $\gamma$-Fe (nanoparticles and thin films, ferromagnetic and antiferromagnetic), thereby motivating a theoretical reassessment of the magnetic states of $\gamma$-Fe taking into account fet distortion.

\section{ACKNOWLEDGMENTS}

The authors thank the Fund for Scientific ResearchFlanders, the Concerted Research Action of the KU Leuven (GOA/14/007), the KU Leuven BOF (STRT/14/002), the Hercules Foundation, the Portuguese Foundation for Science and Technology (CERN/FIS-NUC/0004/2015), and the European Union Seventh Framework through ENSAR2 (European Nuclear Science and Applications Research, Project No. 654002), and SPIRIT (Support of Public and Industrial Research Using Ion Beam Technology, Contract No. 227012). We acknowledge the European Synchrotron Radiation Facility (ESRF) for providing beam time (experiments 26-01-1018, 26-01-1057, 20-02-728, HC-1850, HC-2208), as well as C. Baehtz, N. Boudet, and N. Blancand for support during the experiments. We acknowledge the ISOLDE-CERN facility for providing beam time (experiment IS580) and technical assistance. The authors (L.M.C.P., F.K.) acknowledge the facilities and the scientific and technical assistance of the Australian Microscopy \& Microanalysis Research Facility at the Centre for Advanced Microscopy, Australian National University. We also acknowledge the contribution of Prof. Mark Ridgway (Australian National University), who passed away before the work was completed.
[1] M. van Schilfgaarde, I. A. Abrikosov, and B. Johansson, Nature (London) 400, 46 (1999).

[2] F. Decremps and L. Nataf, Phys. Rev. Lett. 92, 157204 (2004).

[3] P. Gorria, D. Martínez-Blanco, J. A. Blanco, A. Hernando, J. S. Garitaonandia, L. F. Barquín, J. Campo, and R. I. Smith, Phys. Rev. B 69, 214421 (2004).

[4] P. Gorria, D. Martínez-Blanco, J. A. Blanco, M. J. Pérez, A. Hernando, L. F. Barquín, and R. I. Smith, Phys. Rev. B 72, 014401 (2005).
[5] S. L. Palacios, R. Iglesias, D. Martínez-Blanco, P. Gorria, M. J. Pérez, J. A. Blanco, A. Hernando, and K. Schwarz, Phys. Rev. B 72, 172401 (2005).

[6] L. Nataf, F. Decremps, M. Gauthier, and B. Canny, Phys. Rev. B 74, 184422 (2006).

[7] P. Gorria, D. Martínez-Blanco, M. J. Pérez, J. A. Blanco, A. Hernando, M. A. Laguna-Marco, D. Haskel, N. Souza-Neto, R. I. Smith, W. G. Marshall, G. Garbarino, M. Mezouar, A. Fernández-Martínez, J. Chaboy, L. Fernandez Barquín, J. A. 
Rodriguez Castrillón, M. Moldovan, J. I. García Alonso, J. Zhang, A. Llobet, and J. S. Jiang, Phys. Rev. B 80, 064421 (2009).

[8] P.-J. Hsu, J. Kügel, J. Kemmer, F. P. Toldin, T. Mauerer, M. Vogt, F. Assaad, and M. Bode, Nat. Commun. 7, 10949 (2016).

[9] W. Pepperhoff and M. Acet, Constitution and Magnetism of Iron and Its Alloys (Springer, Berlin, 2001), p. 226.

[10] T. Yokoyama and K. Eguchi, Phys. Rev. Lett. 107, 065901 (2011).

[11] M. L. Winterrose, M. S. Lucas, A. F. Yue, I. Halevy, L. Mauger, J. A. Munoz, J. Hu, M. Lerche, and B. Fultz, Phys. Rev. Lett. 102, 237202 (2009).

[12] T. Yokoyama and K. Eguchi, Phys. Rev. Lett. 110, 075901 (2013).

[13] C. S. Wang, B. M. Klein, and H. Krakauer, Phys. Rev. Lett. 54, 1852 (1985).

[14] G. L. Krasko, Phys. Rev. B 36, 8565 (1987).

[15] V. L. Moruzzi, Phys. Rev. B 41, 6939 (1990).

[16] E. Sjöstedt and L. Nordström, Phys. Rev. B 66, 014447 (2002).

[17] V. M. García-Suárez, C. M. Newman, C. J. Lambert, J. M. Pruneda, and J. Ferrer, Eur. Phys. J. B 40, 371 (2004).

[18] H. Magnan, D. Chandesris, B. Villette, O. Heckmann, and J. Lecante, Phys. Rev. Lett. 67, 859 (1991).

[19] J. Thomassen, F. May, B. Feldmann, M. Wuttig, and H. Ibach, Phys. Rev. Lett. 69, 3831 (1992).

[20] J. H. Dunn, D. Arvanitis, and N. Mårtensson, Phys. Rev. B 54, R11157 (1996).

[21] D. Schmitz, C. Charton, A. Scholl, C. Carbone, and W. Eberhardt, Phys. Rev. B 59, 4327 (1999).

[22] P. Ohresser, J. Shen, J. Barthel, M. Zheng, C. V. Mohan, M. Klaua, and J. Kirschner, Phys. Rev. B 59, 3696 (1999).

[23] H. Mühlbauer, C. Müller, and G. Dumpich, Philos. Mag. B 80, 227 (2000).

[24] D. Spisák and J. Hafner, Phys. Rev. Lett. 88, 056101 (2002).

[25] S. Abrahams, L. Guttman, and J. Kasper, Phys. Rev. 127, 2052 (1962).

[26] G. Johanson, M. McGirr, and D. Wheeler, Phys. Rev. B 1, 3208 (1970).

[27] Y. Tsunoda, S. Imada, and N. Kunitomi, J. Phys. F: Met. Phys. 18, 1421 (1988)

[28] S. H. Baker, A. M. Asaduzzaman, M. Roy, S. J. Gurman, C. Binns, J. A. Blackman, and Y. Xie, Phys. Rev. B 78, 014422 (2008).

[29] K. Haneda, Z. X. Zhou, A. H. Morrish, T. Majima, and T. Miyahara, Phys. Rev. B 46, 13832 (1992).

[30] S. H. Baker, M. Roy, S. C. Thornton, and C. Binns, J. Phys.: Condens. Matter 24, 176001 (2012).
[31] J. F. Ziegler, M. D. Ziegler, and J. P. Biersack, Nucl. Instr. Meth. Phys. Res. Sect. B 268, 1818 (2010).

[32] H. Hofsäss and G. Lindner, Phys. Rep. 201, 121 (1991).

[33] U. Wahl, J. G. Correia, S. Cardoso, J. G. Marques, A. Vantomme, G. Langouche, and ISOLDE Collaboration, Nucl. Instrum. Meth. Phys. Res. B 136-138, 744 (1998).

[34] M. Newville, J. Synchrotron Radiat. 8, 322 (2001).

[35] B. Ravel and M. Newville, J. Synchrotron Radiat. 12, 537 (2005).

[36] A. L. Ankudinov, B. Ravel, J. J. Rehr, and S. D. Conradson, Phys. Rev. B 58, 7565 (1998)

[37] L. M. C. Pereira, J. P. Araújo, M. J. Van Bael, K. Temst, and A. Vantomme, J. Phys. D: Appl. Phys. 44, 215001 (2011).

[38] G. Weyer, Mössbauer Effect Methodology (Plenum Press, New York, 1976), p. 301.

[39] V. Augustyns, M. Trekels, H. P. Gunnlaugsson, H. Masenda, K. Temst, A. Vantomme, and L. M. C. Pereira, Rev. Sci. Instrum. 88, 053901 (2017).

[40] H. P. Gunnlaugsson, Hyper. Int. 237, 79 (2016)

[41] L. M. C. Pereira, U. Wahl, J. G. Correia, M. J. Van Bael, K. Temst, A. Vantomme, and J. P. Araújo, J. Phys.: Condens. Matter 25, 416001 (2013).

[42] See Supplemental Material at http://link.aps.org/supplemental/ 10.1103/PhysRevB.96.174410 for a more detailed description of some of the experimental techniques and the corresponding data analysis, which includes Refs. [34-37, 40, 41, 44-47, 53].

[43] J. M. Coey, Magnetism and Magnetic Materials (Cambridge University Press, Cambridge, UK, 2010).

[44] L. H. Bennett and L. J. Swartzendruber, Acta Metall. 18, 485 (1970).

[45] S. J. Campbell and P. E. Clark, J. Phys. F: Metal Phys. 4, 1073 (1974).

[46] W. Keune, R. Halbauer, U. Gonser, J. Lauer, and D. L. Williamson, J. Appl. Phys. 48, 2976 (1977).

[47] W. A. A. Macedo and W. Keune, Phys. Rev. Lett. 61, 475 (1988).

[48] R. J. Weiss, Proc. Phys. Soc. 82, 281 (1963).

[49] S. Oyarzún, A. Tamion, F. Tournus, V. Dupuis, and M. Hillenkamp, Sci. Rep. 5, 14749 (2015).

[50] U. Gradmann and H. Isbert, J. Magn. Magn. Mater. 15-18, 1109 (1980).

[51] S. Mitani, A. Kida, and M. Matsui, J. Magn. Magn. Mater. 126, 76 (1993).

[52] S. V. Okatov, Y. N. Gornostyrev, A. I. Lichtenstein, and M. I. Katsnelson, Phys. Rev. B 84, 214422 (2011).

[53] H. P. Gunnlaugsson, A. Zenkevich, and G. Weyer, J. Alloys Compd. 398, 33 (2005). 